\title{
Open Graph Interface for Uniform Access to E-learning Resources
}

\author{
Meet Malde \\ Dept. Computer Engineering \\ K. J. Somaiya College of \\ Engineering \\ Mumbai, India
}

\author{
Maulik Kothari \\ Dept. Computer Engineering \\ K. J. Somaiya College of \\ Engineering \\ Mumbai, India
}

\author{
Manthan Shah \\ Dept. Computer Engineering \\ K. J. Somaiya College of \\ Engineering \\ Mumbai, India
}

\begin{abstract}
The proposed model provides a method for uniform access to the distributed e-learning resources of the educational institutions and other e-learning providers, to facilitate the developers to build powerful e-learning apps for any platform. The institutions need not provide their own web platform for e-learning, just access to its resources with Open Graph Protocol in JSON format to make it simple for the educationists to build powerful e-learning apps.
\end{abstract}

\section{Keywords}

Open Graph, e-learning

\section{INTRODUCTION}

As the technologies of the 21 st century evolve and mature, we become the beneficiaries of exciting approaches for designing learning experiences. The convergence of number of elearning programs and websites, combined with the explosion of smartphone and tablet use, is having a huge impact on how we think about training and education. The proliferation of smartphones, tablet computers, eBooks, other technology tools, and high-speed Internet access has given students the ability to access high quality learning materials at no $\operatorname{cost}^{[1]}$. Also e-learning removes the age restriction on a human's intelligence. But the educational resources are spread across the whole web in an inconsistent manner i.e. in different forms with each website. Moreover, these resources are available for free and open-access, but they generally don't provide an API to access these resources for other platforms and applications. Some, although do provide API access, like Khan Academy ${ }^{[2]}$, but is not standardized. As these resources are meant to be open and free, the access should be standardized, so that seamless development of applications is achieved. In order to achieve this, we propose Open Graph ${ }^{[3]}$ Interface. The Graph consists of nodes as objects or entities that represent e-learning resources or terms; links between represent the relation or action between the nodes. The Open Graph gives a natural representation of the relationships between the resources and hence makes it easier to understand the data and meta-data. Also, some e-learning providers might want user authentication for various reasons like maintaining points, scores or age restriction, access to some content only to paid users, etc. OAuth 2.0 standard can be effectively used to manage this kind of authentication and authorization.

\section{CURRENT SYSTEMS}

Currently, many e-learning programs of various universities like MIT's OpenCourseWare, Stanford's OpenClassroom, etc. and other pure-web-based e-learning sites like Khan academy, courser, udacity, etc, which also have grading system, are boosting the e-learning trend. However, some don't have APIs while others have different, non-uniform APIs.

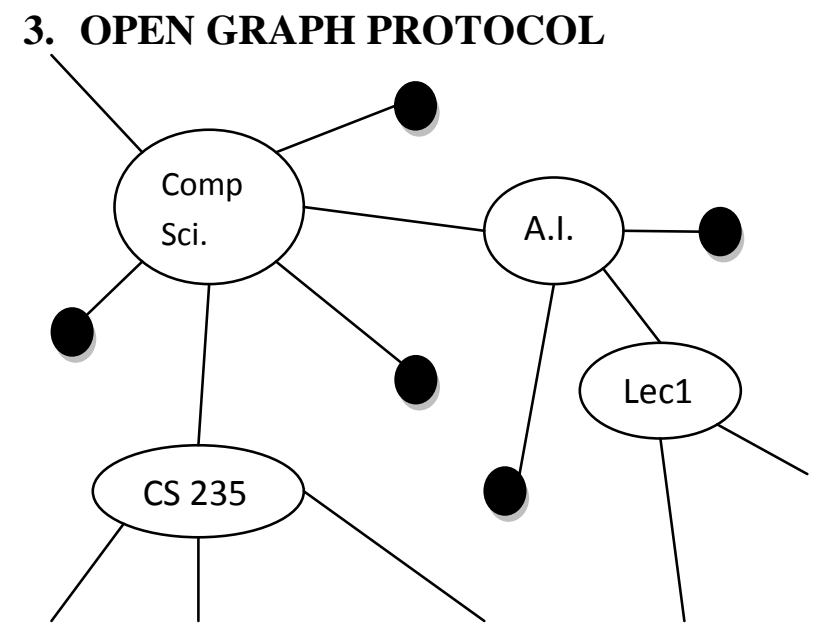

Figure 1

Open Graph provides a simple and effective method of organizing data objects and linking relations between them in the form of a graph. It gives an abstract representation of the objects and relation between the objects in a graphical manner where the nodes of the graph represent objects and the links between nodes represent some relation or action. In the above figure 1, the Comp Sci., a department, represented by a node, is linked to subjects of that particular department which are in turn linked to resources like text, audio or video notes for sub topics.

All these objects and their related data can be retrieved in a particular format by making RESTful ${ }^{[9]}$ API calls vis HTTP in the following manner: url/object-level1/.../object-leveln

Eg: og-hostname/compsci/ai

The e-learning providers, if make their resources available in this protocol format, then the developers can develop intelligent apps that can take relevant information from multiple providers and fill in the shortcomings of single providers or maybe enhance the knowledge/learning provided by a single provider. 
The Open Graph Protocol ${ }^{[3]}$ used and widely publicized by Facebook ${ }^{[4]}$ is actually meant for enabling web pages to become rich objects in its social graph. This, as specified in the Open Graph Protocol specification ${ }^{[3]}$, enriches the web pages with a lot of semantic and meta-data in XML format. For e-learning purpose, the objects are educational resources like text documents and notes, audio and video lectures, etc. and not just web pages. So, for representing data in the objects, JSON (JavaScript Object Notation) ${ }^{[5]}$ format is optimal as it is not bound to XML-like representation and hence fat-free ${ }^{[6]}$. The terms and their implementations are discussed in-depth in the next sections.

\section{METHODOLOGY}

Figure 2 shows the proposed architecture for e-learning. To keep the current infrastructure of the e-learning providers intact and also in line with the Open Graph protocol, two additional modules need to be added to their current systems. The e-learning providers, generally, have their data and other resources stored on public or private cloud. To give the Open Graph API access, an abstraction layer should be developed to give abstract graph representation of the resources to the developers and outside world. This layer should take request, verify, query the related resources on its own cloud and return the data back to the requested client.

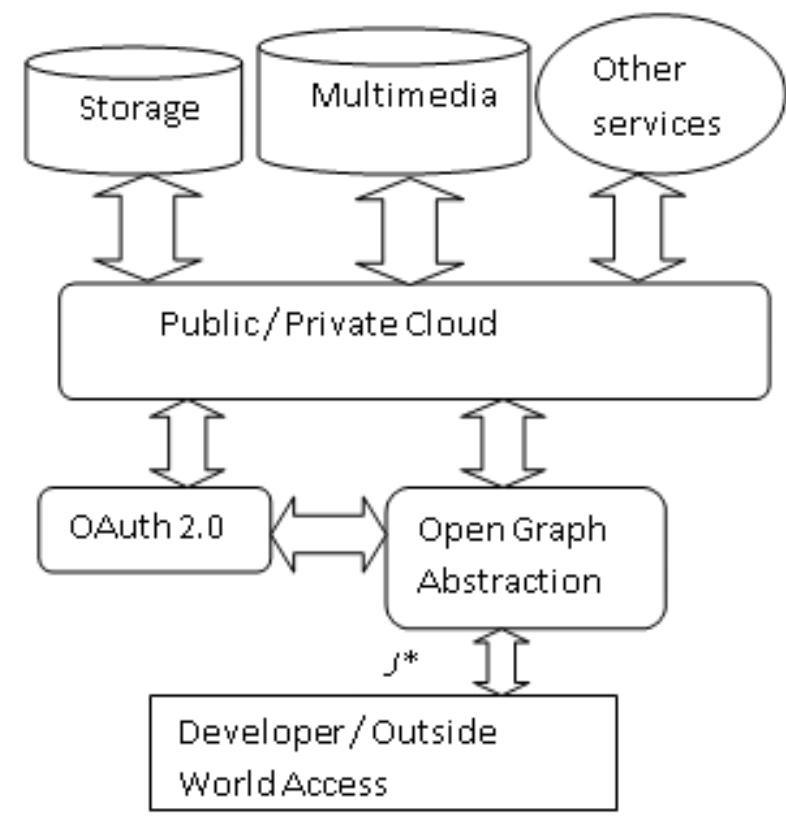

Figure 2

$J^{*}=>$ Data in JSON Format

The data returned back to the client should be in JSON ${ }^{[5]}$ format. For authentication, a separate and optional module for OAuth 2.0 $0^{[7]}$ needs be developed. This manages access control to restricted content. The various terms and techniques are discussed in the following subsections in detail.

\subsection{Open Graph Abstraction Layer}

The main function of this layer is to transform the e-learning resources and other objects that are to be provided for API access, into Open Graph format. For this, the systems schema has to be studied and a new schema, i.e. consistent and uniform for same subject with standardized naming conventions for all providers has to be developed. The resources and related data needs to be stored in this schema format, in databases. NoSQL ${ }^{[9]}$ databases are suggested for storing such data as the variables/attributes may add-up for additional information and many schema-less database use JSON as the document notation ${ }^{[8]}$.

The apps need to make restful API calls and the Open Graph Abstraction Layer verifies, queries and responds back with the data in JSON format. Based on the type of data received, the app may or may not make more calls.

\subsection{JSON Data Format}

The JSON ${ }^{[5]}$ is lighter than XML, i.e. it can represent much more data in less memory. Example of JSON for object Comp $\mathrm{Sci}$. for the figure 1 is

\{

$$
\begin{aligned}
& \text { “dept” : “comp sci.”, } \\
& \text { “related field" : “computers", } \\
& \text { “dept started on” : “1970/03”, } \\
& \text { “courses" : [ [“"name”:"AI”, } \\
& \text { “num":”CS14", } \\
& \text { "con_by":"ABC"\}, } \\
& \text { \{“name":"TCS", } \\
& \text { “num":"C16", } \\
& \text { “con_by":”XYZ”\}] }
\end{aligned}
$$

\}

The e-learning objects can include more detailed data in future and JSON provides that flexibility to include new data easily. Also, JSON is easy to serialize and parse and most of the programming languages have open, free libraries for the same.

\subsection{OAuth 2.0 Authorization module}

OAuth $2.0^{[7]}$ allows for exciting extra functionality and synergies when taking advantage of the social graph and other data and features made available by the OAuth provider. OAuth 2.0 removes the complexity of the server-client signing and password exchange by use of tokens ${ }^{[8]}$. The tokens can be anytime granted and revoked by the server as well as the user. A separate authorization module also makes the system less complex.

\section{USES AND ADVANTAGES}

Currently, there are very few apps for e-learning purposes. But as the app market is growing worldwide and many people now have smartphones or other devices that support apps, the market and users for e-learning apps will surely increase. So to serve these apps, the resources we have at hand should be easily accessible, organized and uniform so that integration of more data from different sites will provide more option and better understanding and knowledge. Also, as Open Graph has to be implemented as a restful services, developers are not restricted to browsers and hence can develop apps for different platform. The below figure 3 shows these advantages 


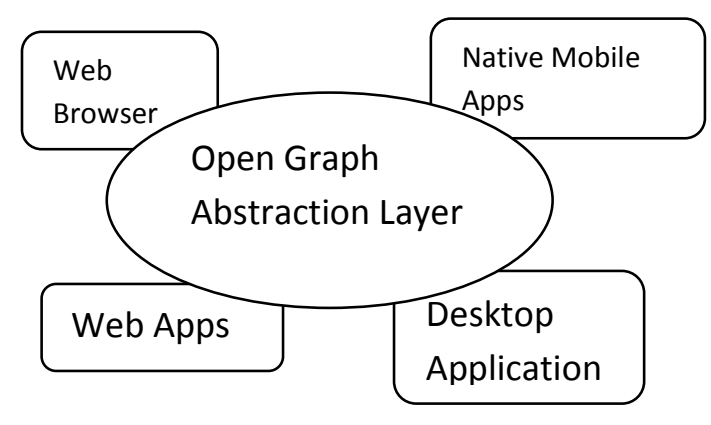

Figure 3

As an example, an application can provide all videos related to Artificial Intelligence course from all e-learning providers on a single page and users can view, rate and comment on those and hence help the community with great content. Also, most e-learning web sites are built with the aim to provide free, unbiased and better education to the people, this concept of uniform access via Open Graph will help serve the goal better.

\section{ACKNOWLEDGMENTS}

We are very much grateful to Department of Computer Science to give us opportunity to work on e-learning platform. We sincerely express our gratitude to Prof. Deepak $\mathrm{H}$ Sharma and Prof. Gajanan Bherde for providing great insight in the field of e-Learning and giving constant inspiration to carry out research work.

\section{REFERENCES}

[1] Welsh, Elizabeth T. and Wanberg, Connie R. and Brown, Kenneth G. and Simmering, Marcia J, "E-learning: emerging uses, empirical results and future directions", International Journal of Training and Development, 245258, Vol. 7, 2003

[2] Khan, S. (2012). The Khan academy. Salman Khan.

[3] The Open Graph Protocol. (2012, July 05). Retrieved Oct 12, 2012, from http://ogp.me

[4] Open Graph - Facebook Developers. (2012, September). Retrieved Oct 12, 2012, from http://developers.facebook.com/docs/opengraph/

[5] D. Crockford. JSON Internet Engineering Tasks Force RFC. (2006, July). http://tools.ietf.org/html/rfc4627

[6] Crockford, Douglas. "JSON: The fat-free alternative to XML." Proc. of XML. Vol. 2006. 2006.

[7] Hammer-Lahav, E., Recordon, D., \& Hardt, D. (2011) The OAuth 2.0 authorization protocol. draft-ietf-oauthv2-18, 8 .

[8] Jones, M. and Hardt, D. and Recordon, D. (2011, June). The Oauth 2.0 protocol: Bearer tokens. Self.

[9] Sutinen, O. (2010). NoSQL-Factors Supporting the Adoption of Non-Relational Databases. http://tutkielmat.uta.fi/pdf/gradu04709.pdf.

[10] Stirbu, Vlad. "Towards a restful plug and play experience in the web of things." Semantic computing, 2008 IEEE international conference on. IEEE, 2008 